\title{
Ramener la justice sociale au centre de la carte : propositions pour un renouvellement critique de la cartographie participative axée sur l'empowerment
}

\author{
Jennifer Barella \\ Institute of Geography, University of Neuchâtel, Neuchâtel, 2000, Switzerland \\ Correspondence: Jennifer Barella (jennifer.barella@unine.ch)
}

Received: 8 August 2019 - Revised: 28 July 2020 - Accepted: 31 July 2020 - Published: 4 September 2020

\begin{abstract}
Résumé. This paper discusses the need for a deeper critical interrogation of participatory mapping (PM) method as a tool for social justice. This stance is informed by the author's involvement in a NGO and community-led PM project in an informal settlement in Khayelitsha (Cape Town, South Africa). The paper argues that academic PM literature is ill-equipped to truly examine its potential for social justice. Firstly, this is due to the PM empowerment framework having shifted from an emancipatory aim to a governing tool. Secondly, this shift does not allow for the consideration of the power relations inherent to PM to be engaged with. This paper concludes by engaging the three epistemological and postcolonial roots of PM in order to provide a starting point for (re)centering PM on social justice.
\end{abstract}

\section{Introduction}

La question de l'empowerment lié à l'utilisation d'informations géographiques produites de façon participative est de plus en plus importante dans tous les pays qui mobilisent ces technologies en tant que levier de développement urbain et outil pour la justice sociale.

Dans les villes du Sud global une grande partie de la population urbaine vit dans des conditions d'habitat précaire et de marginalisation. Celles-ci sont notamment caractérisées par un manque d'accès aux principaux services, une forte vulnérabilité face aux risques naturels, aux expulsions, aux délocalisations forcées et aux insuffisances des programmes de logement social. Ceci est lié avant tout à un manque de reconnaissance politique de la légitimité du « droit à la ville» de ces populations, mais également à un manque de connaissances stratégiques et de données concernant leurs conditions de vie (Parnell and Pieterse, 2014 ; Roy, 2011). Les initiatives qui visent à l'amélioration des conditions de vie de ces habitant.es précaires et à leur reconnaissance politique se heurtent en effet, presque systématiquement, au manque d'informations sur ces lieux, ces populations et leurs dynamiques. De ce fait, la production participative de l'information géographique à l'aide d' outils cartographiques et de Systèmes d'Informations Géographiques (SIG) constitue un moyen privi- légié pour exprimer des revendications territoriales et de développement (Appadurai, 2000; Elwood, 2008).

On assiste aujourd'hui à une diffusion importante et à une standardisation de ces méthodes à l'échelle internationale. Mais, que reste-t-il en terme d'empowerment, en particulier lorsque les objectifs de ces projets ne sont pas atteints ou lorsque les projets tombent dans une impasse ?

Pour répondre à cette question, l'article partira d'une expérience de cartographie participative menée en 2018 dans la ville du Cap, en Afrique du Sud. La première section présentera la mise en œuvre de ce projet en insistant sur les raisons qui ont conduit à son apparent échec. En effet, le plan d'aménagement conçu sur la base de cette cartographie ne verra vraisemblablement pas le jour. Cependant, d'autres dynamiques sur le plan à la fois social et politique sont apparues au cours de cette expérience. L'échec du projet est donc peut-être plus lié à un mauvais ajustement de la grille d'analyse qu'à une inefficacité de l'opération de cartographie en elle-même. La suite de l'article explore alors de manière critique les outils conceptuels autour de la notion d'empowerment que la méthode « cartographie participative » permet de mettre en évidence, à la fois de manière théorique et opérationnelle. Afin de réconcilier la cartographie participative avec une approche plus critique des jeux de pouvoir qui la sous-tendent, la seconde section de l'article reviendra sur l'histoire de cette pratique - du Participatory 
Rural Appraisal dans le Sud global à l'aménagement participatif dans le Nord global - et montrera que l'analyse du potentiel émancipatoire de ces pratiques a été progressivement abandonnée en faveur de leur emploi comme outil de gestion et de « gouvernance néolibérale» (Bacqué et Biewener, 2013). Pour finir, l'article retracera les enjeux émancipatoires des méthodes participatives de cartographie qui peuvent être retrouvées dans trois sources épistémologiques - la critique de la raison cartographique, la critique de l'Etat développementaliste et les approches STS postcoloniales et convergent vers la recherche de justice sociale par le biais de la critique postcoloniale.

\section{L'échec d'un projet de cartographie participative à Khayelitsha (Afrique du Sud), problèmes de méthode ou biais d'analyse ?}

Dans le cadre de la recherche-action à laquelle j'ai participé en 2018, j'ai travaillé pendant cinq mois pour Development Action Group (DAG), une organisation non gouvernementale (ONG) basée dans la ville du Cap en Afrique du Sud. Depuis 1986, et donc bien avant la fin de l'apartheid, l'ONG soutient les collectivités locales dans leurs revendications de droits politiques et spatiaux, notamment au moyen de programmes de formation en leadership, d'aide au logement social et de développement des quartiers informels. Un de ses slogans met la dimension émancipatoire au centre «Empowering people to create change since $1986 »$.

Mon rôle au sein de DAG était d'aider à la mise en place d'un projet pilote de cartographie et de recensement participatifs dans le quartier informel nommé « S section» dans le township de Khayelitsha. Plus, précisément, j’étais en charge de digitaliser la collecte des données spatiales et sociodémographiques au moyen d'applications SIG de collecte de géodonnées, ainsi que d'animer les ateliers de formation pour les cartographes volontaires de $\mathrm{S}$ section et de les accompagner dans les étapes cartographiques et de recensement. Cette participation active m'a permis de faire simultanément de l'observation participante et de mener des entretiens semi-directifs avec divers acteurs locaux - avec les membres d'ONGs travaillant dans le même secteur que DAG, avec des habitant.es de S section ainsi qu'avec des fonctionnaires du gouvernement de la municipalité du Cap et de la province du Western Cape.

La communauté de S section est composée d'environ 700 habitant.es. Depuis plusieurs décennies, elle est installée sur une surface qui s'étale sur trois parcelles cadastrales différentes. A l'époque du projet, elles appartenaient toutes à la municipalité du Cap, mais n'étaient pas toutes légalement constructibles. Le quartier, hérité de la ségrégation urbaine issue de l'apartheid, est entouré par des habitations formelles et dépend politiquement et fonctionnellement de l'aire métropolitaine du Cap. Comme de nombreuses autres poches de pauvreté urbaine, il est caractérisé par des structures d'habi- tat et d'installations sanitaires précaires et insuffisantes pour garantir des conditions de vie saines et dignes pour les résident.es - au sens des objectifs d'Habitat III (UNHabitat, 2017) - ainsi que par une forte pauvreté, de nombreux problèmes de sécurité et un taux de chômage élevé.

Comme de nombreux projets de cartographie participative, le travail s'est déroulé en deux étapes principales, qui visaient à récolter des données spatiales d'une part et socioéconomiques d'autre part. La première étape consistait à cartographier les maisons informelles (shacks) et les infrastructures sur le terrain, par une comparaison d'images aériennes avec l'existant, en se rendant sur place. Une cartographie manuelle était réalisée à partir de relevés de terrain, puis les informations étaient numérisées à l'aide de SIG. La deuxième étape était celle d'un recensement des résident.es au moyen d'un questionnaire sociodémographique élaboré ad hoc par l'ONG en collaboration avec les résident.es. Ce dernier était rendu disponible au moyen d'une application pour tablettes et smartphones, et également réalisé sur la base de SIG.

Ce protocole de recherche a permis la production d'une représentation cartographique du quartier, le recensement des aménités et services publics, ainsi que la création d'un registre des habitant.es et de leurs conditions financières et d'emploi. Ce profiling était nécessaire afin d'établir une image de base de $\mathrm{S}$ section, de repérer qui était potentiellement bénéficiaire de subventions gouvernementales pour le logement ${ }^{1}$ et de documenter les secteurs lacunaires en matière d'investissements publics. S'appuyant sur cet état des lieux cartographique, l'objectif premier du projet était de soutenir les revendications d'accès au logement social et à la propriété foncière des résident.es de $\mathrm{S}$ section face à la municipalité du Cap. Le but du projet était donc d'aboutir à un empowerment de la communauté par le biais de l'acquisition de droits fonciers et de meilleures conditions de logement - un empowerment distributif au sens de la littérature sur la cartographie participative (Elwood, 2002). Pour ce faire, le processus participatif a débouché sur l'élaboration d'un plan de développement intégré du quartier permettant à la fois de reloger chaque résident.e in situ, d'aménager de l'espace public et de respecter les quotas d'accès aux typologies de logement gouvernemental. Sur la base du plan, il

1. En Afrique du Sud, depuis l'entrée en vigueur du cadre de politiques socio-économiques du Reconstruction and Development Programme (RDP) postapartheid, les foyers disposant d'un faible revenu ont le droit d'accéder à une propriété ou à des logements fournis par le gouvernement. Le type de logement auquel les foyers auront accès dépend de leur niveau de revenu. Cependant, dans la pratique, le droit au logement des citoyen.nes sud-africain.es les plus démuni.es se traduit souvent par une longue attente, exacerbant les difficultés de leurs conditions de vie (Oldfield and Greyling, 2015). Les foyers tendent donc à trouver des solutions alternatives pour gagner en visibilité et accélérer les interventions sur leur cadre de vie. Comme pour S section, s'organiser au niveau social en demandant l'appui d'ONG locales est une stratégie couramment employée dans la région du Cap. 
était envisagé de tenir la municipalité responsable de ses politiques d'aménagement et de rendre prioritaire l'intervention urbaine à $\mathrm{S}$ section. Cependant, les négociations se sont rapidement trouvées dans une impasse à cause de l'impossibilité de trouver un accord entre les acteurs - le leadership de S section, l'ONG et la municipalité du Cap. Aggravé par l'incapacité de la communauté locale elle-même à s'entendre en interne, ce blocage a finalement amené l'ONG à abandonner le projet - comme l'explique rétrospectivement un collaborateur de l'ONG «one of the reasons why we've actually decided not to pursue the S section thing anymore is because there was no tangible outcome that could happen from this [mapping and enumeration project] » (Entretien personnel, juin 2019). On peut donc se demander ce qu'il reste de l'objectif du projet - «Empowering people to create change », un des slogans de l'ONG - malgré l'échec de la mise en application pratique de ce plan.

La réponse n'est pas évidente. Dans la littérature scientifique existante, les projets de cartographie participative qui ont échoué sont rarement relatés, et leur apport aux collectivités locales en termes d'empowerment reste peu abordé. Les études qui traitent du potentiel d'empowerment de la cartographie participative ne proposent ni n'appliquent une conceptualisation claire de cette notion. Comme le remarque Elwood (2002), ces études n'interrogent pas réellement la diversité des raisons et des conditions dans lesquelles un tel projet aboutit effectivement à des formes d'empowerment des participant.es, et, surtout, elles associent presque systématiquement l'empowerment à l'aboutissement des objectifs des projets. Si l'on reprend cette approche, l'expérience conduite à S section n'aurait pas produit l'empowerment souhaité en raison de l'absence de mise en application des préconisations du plan de développement, et donc en raison de l'absence de réponse concrète aux revendications d'accès au logement. Par ailleurs, aucune réflexion autour de l'idée d'un disempowerment ou d'une instrumentalisation de ces projets n'a été proposée.

En revanche, de tels projets participatifs sont de plus en plus fréquents et exigent des efforts importants - du point de vue individuel, collectif et organisationnel. Ils impliquent un engagement sur le long terme, des investissements parfois onéreux, de nombreuses ressources humaines et techniques, la mise en place de collaborations, l'apprentissage de compétences variées, etc. Inévitablement, les impacts de ces projets sont bien plus importants et immédiats que leur seule visée finale. L'empowerment est incarné dans des acteurs et se manifeste de manières variées. Par exemple, dans le cas de $\mathrm{S}$ section, le projet cartographique a notamment été utilisé par les habitant.es comme espace de formation, de recherche d'emploi, comme plateforme pour gravir les échelons du leadership local, comme espace pour profiter de la technologie à des fins personnelles ou pour obtenir une rétribution, etc. Cet espace a été également utilisé par le leadership du quartier et par certains fonctionnaires des sub-councils pour faire de la propagande politique, pour acquérir des informations sur des tiers, etc. Parallèlement, pour l'ONG ce projet était une opportunité pour tester sa capacité d'innovation, pour former son personnel à l'utilisation des SIG, mais aussi pour créer des coalitions entre ONGs et collectivités informelles, et pour dévoiler les agendas politiques intéressés des politiciens locaux, etc.

Or, sans un recentrement autour de la question de la justice sociale par le biais d'une conceptualisation de l'empowerment (en tant que redistribution de ressources et remise en question des rapports de domination), ces aboutissements et détournements gravitant autour de la cartographie participative ne peuvent pas être restitués ni analysés. Passer à côté de ces observations équivaut à passer sous silence les conditions dans lesquelles les luttes pour une justice sociale et spatiale se négocient par, pour et avec les populations les plus démunies ou marginalisées. Ces limites rencontrées lors d'une expérience de terrain, justifient l'urgence d'interroger davantage le cadrage théorique de ces méthodes.

\section{La cartographie participative et l'empowerment, histoires d'une dépolitisation}

Cette section présente la cartographie participative et la notion d'empowerment, puis aborde la manière dont l'empowerment est traité dans la cartographie participative pour la justice sociale.

\subsection{La cartographie participative, une discipline d'étude et un outil de développement à l'intersection de théories, pratiques et contextes}

La cartographie participative est l'union de la pratique cartographique et des méthodes participatives. Il s'agit d'un outil méthodologique et épistémologique qui vise une transformation sociale par le biais de la production et de l'utilisation collective et partagée d'information spatiale - dans ses formes variées. La cartographie participative revendique une méthodologie inclusive et décentralisée de la production de l'information géographique ${ }^{2}$ (Chambers, 2006). Sous cette appellation générale sont regroupées toute une série de pratiques collectives, allant de l'élaboration de croquis cartographiques, à la récolte de données spatiales pour le profiling des communautés ${ }^{3}$ (Appadurai, 2012 ; Cochrane et al.,

2. Comme le montre Hirt au sujet des cartographies autochtones, les pratiques d'élaboration collective et participative des savoirs géographiques en tant que telles ne sont de loin pas un phénomène récent (Hirt, 2008). Certaines formes particulières uniquement ont été documentées et formalisées en tant que méthodologies et pratiques de la discipline cartographique dans les quatre dernières décennies seulement. C'est de ces dernières pratiques que traite cette publication.

3. Par community profiling on entend une méthode pour établir une image « de base » de la communauté concernée en recueillant un set de données spécifiques sur une série de facteurs variés (Banque Mondiale). 
2014 ; Day et al., 2007), à la création de plateformes web ou d'applications avec des interfaces cartographiques (Hagen, 2017), à l'analyse d'images satellitaires (Dennis et al., 2005; Nora and Niina, 2009), etc. - comme le montre aussi le cas de S section à Khayelitsha. Ces méthodes sont opérationnalisées par de nombreux acteurs - chercheurs et chercheuses universitaires, acteurs étatiques, agents internationaux du développement et de plus en plus d'acteurs non-étatiques comme les ONG - et dans des domaines variés - comme le développement, l'aménagement du territoire, la gestion des risques environnementaux et la gouvernance urbaine.

Afin de comprendre les inégalités socio-spatiales et de trouver une manière d'y répondre, la géographie a largement questionné l'imbrication entre espace et pouvoir - par exemple à travers des travaux de Alain Reynaud (1981) et David Harvey (1973). Puisque justice sociale et spatiale sont intimement liées (Lefebvre, 1974 ; Soja, 2009), la cartographie participative est un bon outil pour renouveler les méthodes de recherche car elle se positionne à l'interface entre pratique et théorie. Avant de devenir un objet et une méthode de recherche, la cartographie participative a vu le jour dans le domaine du développement rural et de la gestion des ressources naturelles dans les pays du Sud global (Chambers, 2007). Les prototypes de ces méthodes sont formalisés par Robert Chambers dans les années 1980 dans le cadre du Participatory Rural Appraisal (PRA). Le PRA regroupe une série de méthodes qui permettent aux participant.es d'agir sur leurs conditions de vie par la production et la valorisation de leurs connaissances sur le territoire afin de répondre à des problématiques qu'elles ont elles-mêmes identifiées. La production et l'utilisation de l'information spatiale, le plus souvent sous forme cartographique, sont un outil central de ces méthodes. Dans les premières expérimentations, cette approche cartographique était utilisée par les expert.es en tant que manière de « débroussailler le terrain d'étude » et de collecter des « informations référentielles sur le territoire » à l'aide des populations qui y habitent (Burini, 2012). Elle a rapidement évolué vers une méthode d'action participative qui permet aux populations les plus démunies ou marginalisées de prendre le processus en main, de revendiquer la connaissance produite et de guider les priorités des projets de développement dans un but d'auto-détermination (Burini, 2012 ; Chambers, 2006). Les sources principales de la cartographie participative en tant que discipline d'étude peuvent être identifiées dans la cartographie critique, les études du développement et, plus récemment les Science and Technology Studies (STS) appliqués à la cartographie et aux SIG (cf. Sect. 4).

La transformation du rapport de pouvoir entre expert.es et populations locales (Burini, 2012) et l'entrée dans l'époque du numérique (Castells, 1999), sont parmi les principes qui ont contribué au succès de ces pratiques. La reconnaissance de l'expertise des individus et des collectifs sur leur propre territoire, introduite par le PRA, - par opposition à la valorisation de l'expertise du chercheur cartographe uniquement - encourage les méthodologies participatives et l'en- gagement des chercheuses et chercheurs dans l'action sociale. Les recompositions politiques majeures engagées dans les décennies précédentes, comme les luttes pour l'indépendance des pays colonisés et les transitions démocratiques, poussent davantage à questionner l'inclusion des citoyen.n.es dans les débats publics et les manières dont leurs revendications peuvent remonter vers la sphère publique du gouvernement. C'est dans ces contextes que Peluso (1995) désigne par le terme « contre-cartographie » (counter-mapping) toute production et utilisation de la cartographie où l'information géographique ne représente pas les intérêts des élites mais exprime les revendications des populations marginalisées en vue d'obtenir des changements sociaux.

L'importante diffusion de la cartographie participative à partir des années 1990 s'explique par deux facteurs convergents concernant les innovations technologiques. Premièrement, les nouvelles technologies de l'information géographique (les SIG, le GPS et les logiciels d'acquisition d'images satellitaires) ont diversifié les formes du savoir géographique et renforcé la propagation de ces pratiques. Par exemple, le champ des SIG participatifs émerge dans la seconde moitié des années 1990 et s'insère entre autres dans la filiation de la cartographie participative (Dunn, 2007; Harris and Weiner, 1998). Le rôle des SIG d'abord et du Geoweb ensuite, qui numérise et permet la diffusion en ligne de données géographiques, ont tout particulièrement façonné les analyses et les applications de ces méthodes (Crampton, 2011). Notamment, depuis les années 2000 les initiatives collaboratives menées par les contributeurs de OpenStreetMap, pour combler les lacunes en matière d'information géographique dans les pays du Sud global, sont également analysées au prisme de la cartographie participative (Pánek, 2011.).

Deuxièmement, la démocratisation des techniques de l'information et de la communication (TIC) - l'accès aux équipements informatiques et à Internet - est présentée par les organisations internationales en tant qu'opportunité pour impulser un développement (Dakouré, 2014) et pour implémenter davantage d'initiatives de cartographie participative. En effet, la production d'information géographique pour représenter les savoirs et les conditions de vie des populations les plus démunies ou marginalisées est devenue l'une des techniques participatives les plus répandues parmi celles élaborées par les agences internationales de développement, puis par les ONG locales, en faveur d'une réduction de la pauvreté dans les pays du Sud global (Burini, 2012).

La cartographie participative a donc connu un essor considérable dans des contextes géographiques divers et au service de projets sociétaux différents. De même, la littérature rassemblée sous la dénomination de « cartographie participative » étudie en réalité différents modes de production des informations spatiales (Chambers, 2006). Les recherches sont largement façonnées par les travaux sur les SIG et notamment par la société du centre de recherche nord-américain NCGIA (National Centre for Geographical Information and Analysis). C'est donc dans le Nord global que ces méthodes 
ont principalement été théorisées - par opposition à leur contexte d'émergence et à leur immense multiplication dans les pays du Sud global (Mukherjee, 2015 ; Radil and Anderson, 2019).

Cette disjonction géographique illustre bien le clivage épistémologique mis en exergue par Jennifer Robinson (2006) qui distingue études urbaines dans le Nord global et études du développement dans le Sud global. La littérature existante sur la cartographie participative se divise en effet en deux catégories : les théories et études de cas portant sur des initiatives de développement dans le Sud global d'une part, et celles issues des études urbaines et de l'aménagement dans le Nord global d'autre part (Brown and Kyttä, 2014 ; Radil and Anderson, 2019). Dans les cas issus du Nord global, la cartographie participative se greffe aux débats autour de la participation dans le cadre de l'aménagement urbain et, plus récemment, dans le champ d'étude des savoirs citoyens (citizen science) (Thompson, 2016). Ces travaux se focalisent davantage sur des expériences institutionnalisées, qui se déploient sous le contrôle de l'autorité politique qui les promeut (Radil and Anderson, 2019). Dans les cas issus du Sud global, en revanche, les premières études datent des approches PRA et étaient focalisées sur la gestion des risques et l'évaluation des vulnérabilités dans des contextes ruraux (Mukherjee, 2015). Enfin, dans les pays du Sud global plusieurs initiatives de cartographie participative sont promues par des organisations non gouvernementales (ONG) ou des groupes d'activistes qui travaillent pour les collectivités locales. $^{4}$

\subsection{La notion d'empowerment, de la perspective émancipatoire à l'outil néolibéral}

La notion d'empowerment devient centrale dans les années 1990 et 2000 et marque de manière importante les études et pratiques de cartographie participative. En revanche, cette notion sera graduellement dépolitisée par son instrumentalisation au sein de politiques de développement et de réduction de la pauvreté.

D'après Bacqué et Biewener (2013), la notion d'empowerment trouve ses racines dans les luttes du mouvement des droits civiques aux Etats-Unis. Elle se diffuse dans les années 1970 par le biais de la société civile et des mouvements militants féministes, antiracistes et postcoloniaux aux Etats-Unis et en Asie du Sud en particulier - notamment par les travaux de Barbara Bryant Solomon

4. Cette rupture entre contextes géographiques s'applique moins à la littérature sur les cartographies en contexte autochtone. D'une part, par peuples autochtones la littérature se réfère à une catégorie politique et juridique élaborée au sein des Nations Unies et qui s'écarte de la distinction entre Nord et Sud globaux (Hirt, 2008; Lerch, 2014). D'autre part, cette littérature demeure plus ancrée, non sans controverses, dans les débats sur la décolonisation et sur les interdépendances territoriales néocoloniales (Hirt and Lerch, 2013).
(1987) et Lorraine Gutiérrez (1990). Elle est ensuite adoptée par les universitaires pour identifier de nouvelles approches en rupture « avec des modalités d'intervention considérées comme paternalistes, hiérarchiques et inégalitaires » (Bacqué and Biewener, 2013 : 8). Cette rupture s'accompagne d'un renouveau des méthodes de recherche, qui se veulent participatives afin de permettre un engagement explicite entre recherche et action sociale (Schurr and Segebart, 2012). Dans ce même tournant, la cartographie participative s'affirme en tant que processus participatif de recueil de données et de recherche-action.

L'empowerment est à la fois une approche qui a une visée de justice sociale, et un cadre théorique qui étudie les modalités pour y parvenir. Par empowerment on désigne à la fois un état et un processus d'accroissement des capacités d'un individu ou d'un collectif à s'autodéterminer et agir sur sa vie (Sen, 1999). Le terme indique une approche du développement et du changement social, et s'applique au niveau individuel, collectif et politique (Abbott, 2003; Alkire, 2001 ; Friedmann, 1992 ; Zimmerman, 2000). Le niveau individuel, ou psychologique, est le plus largement étudié, notamment grâce aux contributions de la psychologie communautaire (Zimmerman, 2000). Il fait référence aux compétences intrapersonnelles, comportementales et cognitives d'un individu - comme la perception de soi, les compétences, la capacité de comprendre les intentions d'autrui et la volonté d'exercer un contrôle sur sa vie - et se focalise sur la construction d'une « conscience critique » et d'une capacité d'action autonome et programmatique (Gutiérrez, 1990). Le niveau collectif, ou organisationnel, représente la dimension interpersonnelle de l'action et désigne la capacité de « s'organiser avec » et « d'agir sur » - ce qui s'appose à la fois aux individus (création d'un collectif) et aux groupes sociaux partageant des objectifs (coalition). Enfin, le niveau politique, ou social, relève de la transformation de la société dans son ensemble par le biais de l'action collective (Bacqué and Biewener, 2013). Ces niveaux sont distingués dans un but analytique, mais opèrent de manière interdépendante.

La notion d'empowerment postule que les problèmes sociaux et politiques découlent d'une répartition inégale des ressources et de relations de domination à et entre ces niveaux. Pour remédier à ce problème, une démarche d'empowerment passe donc par la redistribution des ressources - qu'elles soient matérielles, symboliques ou de pouvoir - et par l'acquisition de compétences, à travers la participation à des activités et actions collectives (Bénit-Gbaffou, 2015 ; Zimmerman, 2000). L'empowerment ne désigne pas uniquement une transformation individuelle ou collective, mais implique la remise en question des subjectivités et des relations de pouvoir qui sont la cause du différentiel de capacité à s'autodéterminer de certains individus et groupes sociaux (Bacqué and Biewener, 2013). En effet, la notion d'empowerment puise dans la conception poststructuraliste et relationnelle de « pouvoir », tel qu'il est défini à partir des œuvres de Stephen Lukes (1974) et de Michel Fou- 
cault (1975). D'une part, le pouvoir est pensé et se manifeste dans la dialectique entre des formes institutionnalisées (structure) et des formes internalisées (capacité d'agir). D'autre part, les capacités d'agir des collectivités et des individus, dépendent de leur subjectivité, qui est modelée et située dans des rapports de pouvoir. Les processus d'empowerment passent donc par la valorisation de la subjectivité du groupe, ou de l'individu, dit « marginalisé » en tant qu'affirmation et exercice de son pouvoir (Bacqué and Biewener, 2013). Dans cette acception, empowerment sous-entend la conception d'un pouvoir génératif - au sens qu'il promeut un changement, qu'il peut être acquis et échangé - par opposition à un pouvoir de domination ou d'autorité. Il représente l'échelon analytique manquant entre l'individu et le groupe social, car il se manifeste dans la relation entre acteurs et dans l'acte de s'organiser - puisque cet acte implique des échanges et des transferts (d'opinion, de compétences, d'argent, de responsabilités, d'autorité, etc.).

Cette visée transformative et émancipatrice requiert une pleine conscience des matrices d'oppression sociale comme par exemple le genre, la race et la classe - de ce fait, la notion d'empowerment est inhérente aux débats sur la justice sociale. Ces deux termes sont parfois utilisés de manière interchangeable, cependant la justice sociale est à la fois une visée et une dimension centrale de l'empowerment. Dans un but de justice sociale, l'empowerment est un outil conceptuel et analytique qui permet d'appréhender la complexité des rapports de pouvoir et d'aborder structurellement les inégalités sociales.

Dans les années 1990, à travers les organisations internationales, telles que l'Organisation des Nations Unies et la Banque Mondiale, l'empowerment s'impose dans le vocabulaire courant des politiques publiques internationales et nationales de développement - comme le programme Black Economic Empowerment dans 1'Afrique du Sud postapartheid, ou encore le programme Empowerment Zones aux Etats-Unis. Cette internationalisation de l'empowerment repose sur deux facteurs principaux. Premièrement, sur la standardisation du lexique politico-administratif des grandes institutions quant aux stratégies de réduction de la pauvreté (Cornwall and Brock, 2005). Deuxièmement, sur une réorganisation des pouvoirs locaux et nationaux par une « modernisation » administrative qui « condui[t] à repenser le rôle des administrés et [met] en avant la responsabilité des individus et l'enjeu de la participation » (Bacqué et al., 2005 in Bacqué et Biewener, 2013 : 14) vis-à-vis de la lutte contre les inégalités. Ceci est clairement visible dans la réorganisation politique postapartheid en Afrique du Sud, où la participation représente le pilier de l'exercice de la démocratie dans la législation du gouvernement local (Republic of South Africa, 1998).

Dans cette dernière vague, la notion d'empowerment est mentionnée en tant qu'outil de « bonne gouvernance » et est substituée par de nouveaux termes comme, par exemple, participation, citoyenneté et, justement, gouvernance (Bac- qué and Biewener, 2013). Dans cette utilisation, la notion se rapproche de la logique gestionnaire néolibérale qui réduit l'empowerment à une mesure participant d'un projet de management de la population. D'après Cornwall et Brock (2005) dans les politiques de développement, ceci revient d'une part à responsabiliser les plus démunis de leur situation et, d'autre part, à considérer les individus comme des citoyens génériques abstraits. Or, cette vision masque les rapports sociaux de pouvoir sous-jacents à la subjectivité et à la rationalité des acteurs, qui sont nécessaires pour comprendre les spécificités des revendications des populations marginalisées. La dualité conceptuelle de cette notion, qui permet de répondre aux inégalités à la fois par la redistribution des ressources et la remise en question radicale de rapports de pouvoir et de domination, se voit réduite à la première visée distributive uniquement.

Ce tournant vide la notion d'empowerment de sa posture émancipatoire, car il l'écarte des courants féministe et postcolonial et de leur ancrage dans la justice sociale qui était au cœur de l'acception originelle de ce concept. Rapidement, cette acception distributive de la justice sociale se diffuse parmi les militants et les ONG qui emploient ce terme dans une visée émancipatoire, certes, mais également en tant que passe-partout pour obtenir les fonds des bailleurs internationaux (Hirt and Lerch, 2013) - contribuant ainsi à dépolitiser le concept. Le mot « empowerment » était récurrent dans les slogans et logos de l'ONG sud-africaine avec laquelle le projet cartographique à $\mathrm{S}$ section a été réalisé. Le terme apparaissait de manière très visible sur différents supports de communication : les bannières du site web, les en-têtes du papier à lettre, et même dans les images de profil des groupes Whatsapp - un support couramment utilisé pour coordonner les communications diverses concernant les activités de formation de l'ONG au bénéfice des collectivités. En revanche, son sens et ses implications n'ont jamais été discutés, ni lors d'activités avec les communautés, ni lors de rendez-vous visant à définir la stratégie d'action de l'ONG en interne.

D'un projet radical pour la transformation sociale à un buzzword néolibéral vidé de sens politique (Bacqué et Biewener, 2013 ; Cornwall et Brock, 2005), l'utilisation de la notion d'empowerment a largement mué dans le temps. Ce mouvement sémantique et cette dépolitisation a considérablement impacté les outils méthodologiques et épistémologiques qui empruntent ce terme. C'est notamment le cas de la cartographie participative, une discipline à la croisée entre recherche et activisme social.

\subsection{Empowerment et cartographie participative pour la justice sociale}

Si l'empowerment est un outil conceptuel au service de la justice sociale, la cartographie participative apparait davantage comme un outil méthodologique pour l'atteindre.

La cartographie participative s'est saisie de la notion d'empowerment dans les années 1990 et 2000 de plusieurs 
façons. La notion est tout d'abord mobilisée dans les débats sur les avantages et limites de ces approches pour les participant.es (Craig et al., 2002 ; Harris and Weiner, 1998). Mais elle a été aussi mobilisée pour rendre compte de multiples questions plus spécifiques, comme la question des revendications des droits à la terre dans le cas de la cartographie autochtone (Hirt, 2008), celle de la reconnaissance des problématiques des groupes socialement marginalisés (par exemple Freund et al., 2016 et Townley et al., 2016) ou encore celle des luttes pour la reconnaissance et l'aménagement d'habitats précaires (Abbott, 2003) - comme dans le cas de S section à Khayelitsha.

Dans ses recherches d'inspiration féministe, Sarah Elwood rassemble les travaux sur la cartographie et les SIG participatifs qui traitent de la question d'empowerment et propose une catégorisation multidimensionnelle de ce concept en se basant sur les manières dont il a été utilisé dans la littérature. La mobilisation de la notion est courante dans les années 1990-2000, notamment dans la recherche sur le développement communautaire (community development), le militantisme citoyen et l'organisation collective dans le cadre de politiques d'aménagement urbain dans le Nord global (Elwood, 2002). Elwood dégage trois critères analytiques permettant d'évaluer qualitativement le degré d'empowerment de ces méthodes : la transformation distributive (distributive change), la transformation procédurale (procedural change), et le développement de capacités (capacity building).

Premièrement, la « transformation distributive » indique une vision de l'empowerment focalisée sur les résultats du processus qui sont mesurés en relation à une transformation matérielle. Il s'agit notamment des projets de cartographie participative qui ont pour but premier l'amélioration de l'accès à des biens et services et au déblocage d'opportunités de participation à la prise de décision pour des populations marginalisées. C'est le cas des objectifs primaires du projet de $\mathrm{S}$ section, qui mobilisait les méthodes participatives de cartographie dans le but de recueillir les informations stratégiques nécessaires à l'élaboration d'un plan d'aménagement du quartier informel. L'implémentation de ce plan de développement in situ, était la visée primaire du processus, à travers de laquelle évaluer la réussite du projet.

Deuxièmement, la « transformation procédurale » définit l'empowerment en tant que changement substantiel dans la reconnaissance de quel savoir spatial est considéré légitime dans la prise de décision. Il s'agit par exemple d'études qui portent sur l'utilisation de cartes ou de données spatiales produites par une population afin d'obtenir un contrôle sur les décisions qui concernent le territoire qu'elle habite (Young, 2012). Le savoir ordinaire dans ce sens n'est plus seulement rendu visible, mais devient une ressource légitime pour la gestion d'un territoire. De nombreux travaux sur les savoirs spatiaux autochtones (Indigenous Spatial Knowledge) dans le cadre de revendications territoriales correspondent à cette vision émancipatrice de l'information géographique (Hirt, 2008). Or, dans le cas de S section, cette dimension n'était pas valorisée par le rendu final du projet. Conformément à l'objectif final, et afin d'accélérer les interventions sanitaires et l'attribution des logements sociaux pour $\mathrm{S}$ section, le projet cartographique a mobilisé une information géographique conforme au langage des aménageurs. De ce fait, la sélection du type de savoir à représenter a été largement influencée par l'ONG. Plus que le savoir ordinaire et les récits des expériences de vie et des problèmes quotidiens des habitant.es, c'est le savoir géographique dans sa forme managériale qui a été privilégié.

La troisième dimension de l'empowerment est le « développement de capacités » qui se définit en tant qu'extension de la capacité des citoyen.n.es et des collectivités à agir pour leur auto-détermination (Elwood, 2002). Il s'agit de la dimension la plus explorée car elle repose sur la vaste littérature sur les différentiels de capacité d'action (agency achievement) des individus et des collectivités (Alkire, 2001 : 31). Cette conceptualisation s'éloigne de l'idée selon laquelle la redistribution du pouvoir serait liée à une concession plus ou moins temporaire émanant d'une autorité - comme il est sous-entendu dans la « transformation distributive ». Au contraire, elle permet d'appréhender les individus et les collectivités en tant qu'acteurs stratégiques. Cette dimension est très importante au vu de la grande diffusion de ces pratiques car elle pose la question de savoir ce qui reste aux individus et groupes au-delà de la production d'informations géographiques. Par contre, si elle est considérée indépendamment des autres dimensions, elle participe à la responsabilisation des plus démunis vis-à-vis de leur situation et omet ainsi la toile de rapports de pouvoir et de domination coloniale dans laquelle ces acteurs stratégiques opèrent (Cornwall and Brock, 2005). Dans le cas de S section, des ateliers de formation pour les habitant.es étaient intégrés au processus de cartographie et portaient sur des compétences diverses (utiliser et produire une carte, demander de l'aide sociale, comprendre les niveaux de gouvernement sud-africain et leur fonctionnement, participer à la discussion sur le budget municipal, etc.). Or, bien que nombreux, ces moments étaient traités par l'ONG comme de simples effets secondaires du projet - il est donc difficile de saisir leurs apports réels en termes d'empowerment.

Elwood (2002) montre que les études traitent de ces trois dimensions émancipatoires à l'instar d'aboutissements finaux des projets. Elle suggère de considérer ces dimensions de manière conjointe et dans leurs interrelations afin de construire un cadre analytique pour la cartographie et les SIG participatifs. Cependant, même à travers ce cadrage il est ardu d'analyser comment et dans quelles conditions ces projets peuvent être véritablement empowering et pour qui, car les dimensions individuelles (ou psychologiques), organisationnelles et sociales, et notamment les subjectivités des acteurs et leurs rapports de pouvoir, se trouvent mélangés, voire leurs imbrications dans les différentes dimensions ne sont pas traitées. 
Or, c'est bien au sein des rapports de pouvoir entre acteurs, notamment dans les contextes coloniaux ou de ségrégation, que se définissent les objectifs des projets et donc le type d'émancipation souhaitée, mais également les modalités choisies pour les atteindre (le type d'outil cartographique, les informations qui seront représentées, etc.).

\section{Retour sur les prémisses de la cartographie participative : la critique postcoloniale}

Tout comme pour l'émergence de la notion d'empowerment, les sources épistémologiques de la cartographie participative entretiennent un lien fort avec la critique postcoloniale qui n'est que rarement explicité. Ce lien est retracé ici par le biais des trois origines intellectuelles principales de la cartographie participative qui convergent vers une pratique relativement unifiée - la critique de la « raison cartographique ${ }^{5}$, la critique des études du développement et les approches de construction sociale des technologies - et s'appuie sur des exemples issus du projet sud-africain.

La définition du terme « postcolonial » est largement débattue ${ }^{6}$. Cette entreprise politique et intellectuelle trouve ses origines dans les études littéraires (Bhabha, 1984; Said, 1978). En suivant les définitions de Roy (2016), Hirt (2008) et Anderson (2002), le postcolonial est considéré ici en tant que cadre dans lequel appréhender les phénomènes contemporains sous un angle critique, propositionnel et explicitement inséré dans un débat de justice sociale - au sens de remise en question des rapports de domination. Du point de vue épistémologique, il questionne le problème de l'eurocentrisme concernant tant les savoirs coloniaux que leur héritage (Roy, 2016). Les préoccupations postcoloniales de justice sont intrinsèquement spatiales. Puisque le projet colonial est considéré comme un acte de violence géographique, l'insurrection et les revendications des colonisé.es passent également par une recherche et une restitution de l'espace dont ils/elles ont été dépossédé.es, à la fois matériellement et symboliquement (Jacobs, 1996; Said, 1978). Dans la littérature sur la cartographie participative, ceci revient à identifier et à dénoncer la complicité de la discipline cartographique dans la réalisation de tout projet colonial et par extension de tout projet de domination. Dès leur émergence, les projets de cartographie participative se distinguent par la volonté de penser de manière renouvelée les modalités de production de la connaissance spatiale et en particulier de réfléchir aux condi-

5. Ce terme est emprunté à Farinelli, F. : La crisi della ragione cartografica, Einaudi, 2009. La « raison cartographique» fait référence à l'influence historique de la cartographie et de ses corollaires dans la lecture du monde propre à la culture occidentale - et qui aurait été imposée aux peuples du monde par la colonisation.

6. Nous ne souhaitons pas ici simplifier la complexité de cette entreprise intellectuelle, mais nous nous restreindrons à expliciter ce que nous entendons par « postcolonial» dans notre argument. tions dans lesquelles l'inclusion et l'émancipation des populations marginalisées sont possibles.

\subsection{La critique de la raison cartographique - (in)visibilité cartographique}

La critique de la raison cartographique se base sur la reconnaissance de la cartographie en tant que représentation mimétique (Bhabha, 1984) et en tant que construction d'une configuration historique particulière des relations de pouvoir : la représentation géographique n'est que le dessin d'un dessein. La cartographie participative est une réponse aux critiques de la complicité de la cartographie dans les emprises coloniales et impérialistes (Hirt, 2008 ; Huggan, 1989). La carte du colonisateur était un dispositif de hiérarchisation et de normalisation des territoires et des sujets. Elle était l'outil graphique qui inscrivait la rhétorique coloniale sur un espace considéré comme vide et dont on considère qu'il n'a été approprié par personne. Les approches critiques de la cartographie considèrent la carte en tant que « texte culturel», donc en tant qu'élément linguistique, bien que graphique, au service d'une rhétorique de domination (Harley, 1989; Jacobs, 1996). C'est en acceptant l'interprétation textuelle des cartes qu'il est possible de déconstruire la prétention à la neutralité des conventions cartographiques et de montrer qu'elles sont déterminées par des rapports de pouvoir (Harley, 1989; Jacobs, 1996; Pickles, 2006). De ce fait, il devient possible de délibérément s'approprier la question cartographique et de l'utiliser au bénéfice d'un autre type de programme politique. C'est au cœur de cette réflexion épistémologique que la carte acquiert un pouvoir émancipateur qui permet de subvertir les dynamiques d'exclusion et de domination (Peluso, 1995). Comme elle a servi à la création d'un récit, elle permet aussi de l'inverser et d'en raconter un nouveau. Les projets de cartographie participative dans un but de justice sociale postulent que la visibilité cartographique est au centre de la démarche émancipatoire - être sur la carte, c'est exister et réclamer ses droits sur le « vide cartographique». En revanche, comme le remarquent Choplin et Lozivit (2019) dans leur expérience de cartographie participative dans un quartier précaire de Cotonou (Bénin), les enjeux de la visibilité cartographique sont ambivalents. D'une part, visibilité signifie officialiser les revendications des habitant.es de ces quartiers, d'autre part, elle signifie officialiser l'illégalité de certaines conditions que l'on peut rencontrer.

Ce statut ambigu des entreprises des cartographies participatives appliquées à des organisations territoriales informelles en milieu urbain est bien illustré par l'exemple d'étude du projet à $\mathrm{S}$ section, notamment autour de la question de la cartographie des réseaux d'eau. Le projet incluait en effet un recensement des points d'eau dans le quartier. Or, à l'exception d'environ cinq points d'eau officiels installés par la municipalité et partagés par les 700 habitant.es, l'intégralité des connections au réseau d'eau des habitations informelles étaient illégales. Afin de représenter leurs condi- 
tions de vie avec précision, les cartographes volontaires de $\mathrm{S}$ section ont minutieusement recensé tous les rattachements au réseau et les ont indiqués dans la légende en tant que « private taps » (robinets privés). Mais il a été choisi d'exclure cette information de la carte finale car, au lieu de soutenir les revendications de S section pour une amélioration de leurs conditions de vie, elle aurait localisé et quantifié les détournements illégaux du réseau d'eau officiel. $\mathrm{Si}$, d'une part, la représentation soignée des points d'accès à l'eau permettait de montrer la réalité et la débrouillardise des résident.es, elle aurait, d'autre part, officialisé l'illégalité de leur situation. Puisque dans tout contexte territorial la limite entre informel et illégal est très subtile (Willis, 2020), la visibilité cartographique est une arme à double tranchant. De ce fait, la question de ce que l'on représente, au nom de qui et dans quel but, sont des considérations à discuter au préalable de chaque projet de cartographie participative. Par contre, ce que l'on ne voit pas, c'est qu'il faut être prêts à remettre en question ces décisions en cours de route, comme dans l'exemple des robinets, le consensus entre acteurs et le but des cartes sont temporaires.

Notons enfin que les types d'(in)visibilité qui émergent de ces études en milieu urbain - en l'occurrence, en Afrique du Sud et au Bénin (Choplin and Lozivit, 2019) - peuvent être très différents des enjeux présents dans d'autres contextes. Par exemple, d'après l'expérience de Berthier-Foglar en Amérique du Nord (2008), en contexte amérindien c'est la localisation cartographique de sites considérés comme sacrés qui n'est pas toujours souhaitable pour des raisons de protection des sites. De même, la (in)visibilité cartographique, de par le pouvoir performatif de la carte, joue un rôle important dans l'attribution d'identités spatiales - cartographier c'est aussi prendre le risque d'essentialiser des identités et des récits qui dans la réalité sont en perpétuelle mutation (Desbiens et al., 2020 ; Turnbull, 2000).

\subsection{La critique de l'État développementaliste - instrumentalisation par les acteurs}

La nécessité d'une cartographie alternative trouve aussi son origine dans la reconnaissance du rôle de la cartographie dans l'exercice du pouvoir autoritaire et le maintien de l'appareillage étatique, l'État moderne étant un des principaux acteurs à la fois du projet colonial et des politiques du développement. Dans Seeing like a State, James Scott (1998) montre comment les cartes étatiques participent à la création d'une version générique de la réalité du territoire qui n'est qu'une des représentations possibles parmi une pluralité, car elle cache les réalités locales du terrain en faveur d'une vision lissée et unifiée. Scott montre comment ces techniques du savoir géographique - notamment le cadastre et le plan d'aménagement urbain - sont mobilisées pour maintenir le contrôle sur les populations tout en les privant de la possibilité de s'auto-définir et de faire valoir leurs savoirs et leurs droits sur le territoire. Ces critiques conduisent à la naissance de méthodes d'intervention, comme la cartographie participative, qui permettent une émancipation des populations locales à travers leur capacité de parler pour elles-mêmes (Spivak, 1988). C'est au moyen de cette critique que la cartographie, un outil privilégié du « seeing like a state » (Scott, 1998), commence à être considérée en tant que «weapon of the weak» (Scott, 1985). La critique développementaliste pose la question des acteurs et de leurs programmes. Or, nous avons vu que, très souvent ces pratiques sont implémentées par des acteurs non-étatiques du développement, qui sont les nouveaux médiateurs dans le processus de collecte et d'utilisation de l'information géographique. La critique de Scott doit donc être étendue à ces acteurs, car la mobilisation nonétatique de ces pratiques n'indique pas forcément une réappropriation du savoir spatial de la part de la population. $\mathrm{Au}$ contraire, ces pratiques peuvent être instrumentalisées, plus ou moins volontairement, car imbriquées dans une toile d'agendas politiques.

Dans le projet de S section, la mise en œuvre du processus de cartographie participative avait pour fonction, d'une part de répondre aux revendications de $\mathrm{S}$ section, et d'autre part, de tester la capacité de l'ONG à internaliser totalement le processus cartographique et de recensement par le biais de nouvelles technologies de l'information géographique - afin d'éviter de recourir à des experts externes. Ce deuxième intérêt a impacté le déroulement du projet, qui, par exemple, était itératif, ne suivait pas un protocole précis et a posé des soucis quant à la consolidation des données. Ainsi, l'importance d'étapes clé du processus liées aux rouages de la technologie, comme les phases de test des applications, de vérification des flux de données ou de vérification des données saisies lors du recensement, a été largement sous-estimée. Premièrement, en raison d'un manque d'expertise technologique au sein de l'ONG. Deuxièmement, en raison d'une incompatibilité entre les mécanismes de la technologie et le fonctionnement de l'ONG (Barella, 2020). L'opérationnalisation des systèmes d'informations géographiques requiert l'élaboration d'une stratégie stable sur le long terme, alors que le travail de l'ONG se fait par projets de courte durée et doit répondre aux humeurs politiques et à la disponibilité des collectivités. Il faudrait donc pouvoir questionner dans quelle mesure cette instrumentalisation a impacté, ou pas, l'empowerment des participant.es, tant des habitant.es de $\mathrm{S}$ section que de l'ONG en tant qu'acteur organisationnel, au sens de Zimmerman (2000).

Parmi les raisons du blocage du projet, figure un désaccord entre le leadership de $\mathrm{S}$ section et le ward councillor ${ }^{7}$ :

7. Le ward councillor ou conseiller du gouvernement local est une figure particulière du gouvernement local sud-africain qui est censée faire le lien entre les leaders des communautés locales (community leadership) de son aire administrative (ward) et le conseil de municipalité. Il ou elle représente le canal officiel pour faire remonter les revendications des collectivités vers la municipalité. Cependant, ce rôle est souvent instrumentalisé à des fins personnelles, ce qui rajoute un échelon de complexité dans le processus de re- 
" there was influence from the ward councillor in this process that he wanted to use it as some type of political tool» (Collaborateurs ONG, entretien personnel, juin 2019). Bien que n'étant pas invité, ce dernier participait assidûment aux rencontres de quartier et était particulièrement attentif aux étapes intermédiaires du projet. Il souhaitait s'assurer que son nom apparaisse sur les cartes produites dans la collaboration entre la communauté et l'ONG, afin d'affirmer son autorité sur le quartier. Au premier abord ceci peut paraître anecdotique, mais en réalité cela révèle une partie des rapports politiques et de pouvoir dans le cadre desquels la communauté et l'ONG ont dû constamment négocier leurs objectifs cartographiques pour S section. Soudain, le projet cartographique n'était plus négocié uniquement entre l'ONG et la communauté, mais également avec un représentant politique et une administration municipale complexe (Buire, 2011).

Afin de garantir une visée de justice sociale à ces projets, il est nécessaire de considérer les programmes des acteurs qui y participent et, de ce fait, d'évaluer le potentiel de ces projets de manière plus large - puisqu'il implique un transfert/exercice de pouvoir, l'empowerment nécessite de prendre en compte toute une série d'acteurs, autres que les seuls cartographes, susceptibles de modifier le cours de l'action.

\subsection{Les approches STS postcoloniales - le pouvoir du numérique}

Les approches Science and Technology Studies (STS) postcoloniales étudient comment les technologies et les savoirs fonctionnent et se déploient dans des contextes divers. La critique postcoloniale et les préoccupations en matière de justice sociale se manifestent dans le champ des STS dans les années 2000 avec l'émergence des Postcolonial STS, et en réponse à l'optimisme démesuré du « déterminisme technologique ( (Odendaal, 2010; Turnbull, 2000). Ce champ d'études explore non seulement comment les différentes formes de domination marquées par une dimension (néo)coloniale opèrent dans l'interaction entre technologies et sciences, mais aussi comment cette interaction impacte les revendications de la part des populations qui ont le plus subi, ou subissent encore, des formes de domination. Dans une perspective de comparaison interculturelle, Turnbull (2000) contribue au champ des STS postcoloniales en actualisant la notion de tiers-espace (third space) de Bhabha (1984) et l'idée d' " hybride» qui en dérive : tout système de « technoscience » est un assemblage (ou agencement, au sens deleuzo-guattarien) qui est façonné, à un moment donné et dans des circonstances particulières, par les contributions d'acteurs différents (Turnbull, 2000). De par la relation synergique entre science et technologies qui la caractérise, la cartographie est une technoscience à décoloniser par excellence. Les technologies et les savoirs cartographiques en par-

vendication des droits des collectivités locales (Cirolia et al., 2017; Bénit-Gbaffou et al., 2015). ticulier nécessitent donc de nouveaux outils analytiques qui permettent de laisser la place à de nouveaux récits et de dévoiler la complexité de la négociation qui s'opère lors de la création d'une carte ou d'autres formes de savoir spatial.

Les STS postcoloniales promeuvent donc l'analyse de phénomènes et d'acteurs autrement délaissés (Anderson, 2002) en permettant de comprendre la dialectique relationnelle qui façonne la forme des technologies actuelles de production du savoir géographique. Une des préoccupations principales de cette littérature est de préciser comment la forme même de la technologie influence le type de savoir retenu, son utilisation et son appropriation.

La description du projet de $\mathrm{S}$ section a montré que la cartographie participative regroupe un ensemble varié de pratiques et d'outils, ainsi que de formes de représentation de l'information recueillie. Au niveau de la représentation de l'information, le brouillon de la carte imprimée en format A0 que les cartographes volontaires de S section ont présenté lors d'un atelier de discussion du projet n'a pas fait l'unanimité au sein du leadership. Ceci non pas en raison de son contenu - une cartographie minutieuse des maisons existantes qu'il s'agissait de vérifier collectivement - mais en raison de sa matérialité - le format papier. En effet, la reproche concernait la simplicité du support et le fait qu'on ne puisse pas, avec un simple clic, accéder à une bulle d'informations plus détaillée et zoomer dans l'image. Intuitivement, aux yeux du leadership de S section, la médiation par la technologie et l'interactivité de l'information constituait un élément important inhérent au pouvoir performatif de la carte. On peut donc se poser la question de savoir si une carte numérique permettrait un empowerment différent selon les contextes, en considérant que la vision et les attentes qu'ont les acteurs vis-à-vis de la technologie utilisée influence la réussite du projet.

C'est à l'aune des questionnements des STS postcoloniales que cet exemple s'inscrit dans une logique post- et décoloniale. Pour l'ONG, l'atelier de discussion pouvait se dérouler autour d'une carte « frugale»- pour reprendre le terme d' " innovation frugale » utilisé par Choplin et Lozivit (2019) - et donc sans la mobilisation de technologies numériques particulières. En revanche, l'ONG s'est confrontée à un leadership qui avait des idées précises quant au rôle du numérique en tant que levier politique. Cette vision spécifiquement et stratégiquement technophile était liée à deux constats en particulier : la connaissance d'expériences internationales et locales mobilisant des technologies numériques en tant que vecteur de revendication, et le rôle prépondérant du numérique dans les politiques du gouvernement local du Cap. Au sujet du deuxième point, effectivement, l'administration municipale du Cap promeut une politique axée sur les données et sur le numérique en tant que levier pour la participation citoyenne dans la fabrique urbaine (Ricker and Cinnamon, 2020). Cette politique définit le référentiel quant à la forme que peuvent (ou doivent) prendre les revendications des habitants des quartiers informels afin de contester, 
voire de s'aligner sur, la rationalité de l'acteur gouvernemental censé pourvoir à leurs besoins. De par les discussions qu'il a soulevées, le brouillon de carte de $\mathrm{S}$ section apparaît donc en tant qu'assemblage de bouts de récits et de rationalités d'acteurs différents qui se confrontent et qui contribuent à façonner la forme et le contenu du produit cartographique final, ainsi que son utilisation dans une visée d'émancipation.

De même, le choix des géo-technologies pour recenser les données sociodémographiques de la part de l'ONG peut être analysé en tant qu'enjeu de pouvoir qui n'apparaîtrait pas sans un cadre d'analyse postcolonial. Bien qu'il existe des alternatives en open source, les outils technologiques choisis par l'ONG sont produits par Esri (Environmental Systems Research Institute), le géant étasunien des logiciels SIG. Ceci implique l'acquisition de licences pour les logiciels et la dépendance vis-à-vis de l'entreprise nord-américaine quant au stockage des données en ligne. Tout comme pour les formes du savoir géographique, l'adoption de certaines technologies au détriment d'autres dépend avant tout du pouvoir exercé par les acteurs de l'économie politique de ces logiciels - et il en va de même pour les composantes matérielles nécessaires au déploiement de ces outils (Choplin and Lozivit, 2019). Malgré la grande diffusion de ces technologies dans les pays du Sud global (Dakouré, 2014), c'est depuis le Nord global qu'elles sont développées et diffusées. Sans une approche critique on passerait à côté de ces rapports de dépendance néocoloniaux, qui renforcent et s'ajoutent aux rapports de pouvoir issus du clivage entre théorisation de la cartographie participative dans le Nord global et application importante dans le Sud global (cf. Sect. 3.1).

\section{Conclusion - vers une cartographie participative postcoloniale?}

Cette publication avait pour but de montrer que la littérature sur la cartographie participative doit rétablir son lien avec les préoccupations de justice sociale. En se basant sur une analyse de la littérature et de réflexions issues d'une recherche-action en Afrique du Sud, il s'agissait de montrer l'importance de reposer de façon renouvelée et critique la question du potentiel d'empowerment de ces méthodes.

L'impossibilité de comprendre les impacts du projet mené à $\mathrm{S}$ section au-delà de son aboutissement final est tributaire du glissement de la notion d'empowerment en tant qu'outil conceptuel de la justice sociale à un outil de gouvernance néolibérale. Premièrement, cette contribution montre que ce glissement s'est opéré au moment de la multiplication de ces méthodes par les agents du développement - organisations internationales et organisation non gouvernementales - qui coïncide avec un dénouement du sens politique de la notion d'empowerment. Ce problème est souligné également dans la restitution de la littérature sur la cartographie participative et l'empowerment qui s'articule autour de trois dimensions - appelées distributive, procédurale et développement de capacités - et qui sont appréhendées en tant qu'objectifs finaux des projets. La littérature sur la cartographie participative se focalise davantage sur les modalités de mesure de la transformation sociale que sur les mécanismes qui permettent de la provoquer. De ce fait, elle n'est pas équipée pour appréhender les échecs, les effets inattendus et les détournements hors consensus de ces pratiques participatives. Dans ce contexte, l'analyse du potentiel de justice sociale est réduite à une question de redistribution de ressources en délaissant totalement les rapports de pouvoir qui influencent cette redistribution.

Ensuite, la publication reconnecte la cartographie participative avec ses sources épistémologiques postcoloniales afin de montrer l'importance de considérer les rapports de pouvoir dans l'étude des pratiques cartographiques participatives. Elle identifie trois sources principales de la cartographie participative : la critique de la raison cartographique, la critique de l'État développementaliste et les approches STS postcoloniales. Cette historicisation de l'émergence de la discipline montre qu'elle s'est développée en réponse au besoin de repenser l'impact des méthodes cartographiques dans un but d'émancipation et de remise en question de rapports de domination à plusieurs niveaux. À l'aide de brefs extraits issus de l'expérience sud-africaine, cette section dégage trois thématiques clés de la pratique de la cartographie participative qui ne peuvent pas être considérées en dehors de rapports de pouvoir : la tension entre visibilité et invisibilité cartographique, l'instrumentalisation de ces pratiques par différents acteurs, et l'influence du numérique et de son économie politique sous-jacente.

Cette contribution est une tentative de fournir un nouveau point de départ pour une cartographie participative qui puisse retrouver et revendiquer sa place centrale dans les débats et les pratiques adressant les inégalités spatiales, sociales et politiques. Une cartographie participative postcoloniale serait une cartographie participative qui se donne les moyens théoriques, conceptuels et méthodologiques pour opérer et appréhender ses impacts au sein même des rapports sociaux de pouvoir, en dehors du consensus et au-delà des clivages géographiques réitérés par la littérature. Comme nous le rappelle Achille Mbembe (2006 : 121), la critique postcoloniale est « une pensée qui, à plusieurs égards, croit encore au postulat selon lequel il n'y a de savoir que celui qui vise à transformer le monde ». Ramener la critique postcoloniale au centre de la théorie cartographique n'est pas une étiquette à rajouter ou une fin en soi. C'est une manière de prendre au sérieux l'importance de théoriser en partant de l'intégration de points de vue multiples, de repenser de manière critique les impacts des méthodes de recherche sur les savoir spatiaux et leurs usages, et avant tout, de remettre la justice sociale au centre de la carte.

Disponibilité des données. L'ensemble des données utilisées dans le cadre de ce travail (entretiens, observations, cartes et don- 
nées produites lors du recensement participatif du quartier informel, etc.) n'est pas rendu public en raison du caractère sensible et confidentiel du matériel récolté.

Intérêts concurrents. L'auteur déclare qu'elle n'a aucun conflit d'intérêt.

Remerciements. Je tiens à remercier l'ONG Development Action Group et les résident.es de $\mathrm{S}$ section settlement à Khayelitsha que j'ai eu l'opportunité de soutenir pendant ce travail de recherche participative.

Contrôle par les pairs. This paper was edited by Myriam Houssay-Holzschuch and reviewed by two anonymous referees.

\section{Références}

Abbott, J. : The use of GIS in informal settlement upgrading : Its role and impact on the community and on local government, Habitat Int., 27, 575-593, 2003.

Alkire, S. : Valuing Freedoms : Sen's Capability Approach and Poverty Reduction, New York, Oxford University Press, Oxford, 2001.

Anderson, W. : Introduction : Postcolonial Technoscience, Soc. Stud. Sci., 32, 643-658, 2002.

Appadurai, A. : Grassroots Globalization and the Research Imagination, Public Culture, 12, 1-19, 2000.

Appadurai, A. : Why enumeration counts, Enviro. Urban., 24, 639641, 2012.

Bacqué, M.-H. and Biewener, C. : L'empowerment, une pratique émancipatrice, La Découverte, Paris, 2013.

Bacqué, M.-H., Rey, H., and Sintomer, Y. : Introduction. La démocratie participative, un nouveau paradigme de l'action publique ?, in : Gestion de proximité et démocratie participative, La Découverte, Paris, 2005.

Barella, J. : Strategic and acupunctural GIS implementation within community-oriented organisations : evidence-based insights from a South African participatory action-research for informal settlements upgrading, Cartographica : The International Journal for Geographic Information and Geovisualization, accepted, 2020.

Bénit-Gbaffou, C. e. a. : Popular politics in South African cities : unpacking community participation, HSRC Press, Cape Town, 2015.

Berthier-Foglar, S. : Montagnes mythiques des Indiens des EtatsUnis : le sacré et le juridique, Caliban, 23, 237-244, 2008.

Bhabha, H. : Of Mimicry and Man : The Ambivalence of Colonial Discourse, October, 28, 125-133, https://doi.org/10.2307/778467, 1984.

Brown, G. and Kyttä, M. : Key Issues and Research Priorities for Public Participation GIS (PPGIS) : A Synthesis Based on Empirical Research, Appl. Geogr., 46, 122-136, 2014.

Buire, C. : "Bringing Government Closer to the People" ? The Daily Experience of Sub-councils in Cape Town, J. Asian Afr. Stud., 46, 465-478, 2011.
Burini, F. : Cartographie et participation pour la coopération environnementale : le terrain et la restitution des savoirs traditionnels en Afrique subsaharienne, Ann. Geogr., 687-688, 487-512, 2012.

Castells, M. : Le pouvoir de l'identité. L'ère de l'information, Fayard, Paris, 1999.

Chambers, R. : Participatory Mapping and Geographic Information Systems : Whose Map ? Who is Empowered and Who Disempowered? Who Gains and Who Loses ?, The Electronic Journal of Information Systems In Developing Countries, 25, 1, 1-11, 2006.

Chambers, R. : From PRA to PLA and Pluralism : Practice and Theory, IDS, Brighton, 2007.

Choplin, A. and Lozivit, M. : Mapping a slum : learning from participatory mapping and digital innovation in Cotonou (Benin), CyberGEO, https://doi.org/10.4000/cybergeo.32949, 2019.

Cirolia, L. R., Görgens, T., van Donk, M., Smit, W., and Drimie, S. : Upgrading Informal Settlements in South Africa : Pursuing a partnership-based approach, University of Cape Town Press, Cape Town, 2017.

Cochrane, L., Corbett, J., and Keller, P. : Impact of Community-based and Participatory Mapping, Working Paper, Institute for Studies and Innovation in Community-University Engagement, University of Victoria, https://doi.org/10.13140/RG.2.1.4522.5360, 2014.

Cornwall, A. and Brock, K. : What do buzzwords do for development policy ? a critical look at "participation", "empowerment" and "poverty reduction", Third World Q., 26, 1043-1060, 2005.

Craig, W., Harris, T., and Weiner, D. : Community Participation and Geographical Information Systems, Taylor and Francis, London, 2002.

Crampton, J. W. : Mapping : A Critical Introduction to Cartography and GIS, Wiley, New York, Oxford, 2011.

Dakouré, E. : TIC et développement en Afrique : approche critique d'initiatives et enjeux, Revue française des sciences de l'information et de la communication, 4, 2014.

Day, P., Farenden, C., and Gross, H. : Participative community profiling : a method for community informatics research : maps, networks, and stories, Constructing and sharing memory community informatics, identity and empowerment, edited by : Stillman, L. and Johanson, G., in : Constructing and sharing memory community informatics, identity and empowerment, Cambridge Scholars Publishing, Newcastle, UK, 24-36, 2007.

Dennis, R. A., Mayer, J., Applegate, G., Chokkalingam, U., Colfer, C. J. P., Kurniawan, I., Lachowski, H., Maus, P., Permana, R. P., Ruchiat, Y., Stolle, F., Suyanto, and Tomich, T. P. : Fire, People and Pixels : Linking Social Science and Remote Sensing to Understand Underlying Causes and Impacts of Fires in Indonesia, Human Ecol., 33, 465-504, 2005.

Desbiens, C., Hirt, I., and Collignon, B. : Introduction to the special section on Indigenous spatial capital : Incorporating First Peoples' knowledges, places, and relations into mapping processes : Introduction, The Canadian Geographer / Le Géographe canadien, 64, 4-9, 2020.

Dunn, C. E. : Participatory GIS - a people's GIS ?, Prog. Hum. Geog., 31, 616-637, 2007.

Elwood, S. : GIS Use in Community Planning : A Multidimensional Analysis of Empowerment, Environment and Planning A : Economy and Space, 34, 905-922, 2002. 
Elwood, S. : Volunteered geographic information : key questions, concepts and methods to guide emerging research and practice, GeoJournal, 72, 133-135, 2008.

Farinelli, F. : La crisi della ragione cartografica, Einaudi, 2009.

Foucault, M. : Surveiller et Punir, Gallimard, Paris, 1975.

Freund, L., Hawkins, B., and Saewyc, E. : Reflections on the use of participatory mapping to study everyday health information seeking by LGBTQ youth, Proceedings of the Association for Information Science and Technology, 53, 1-4, 2016.

Friedmann, J. : Empowerment : the politics of alternative development Blackwell, Cambridge, Mass, 1992.

Gutiérrez, L. : Working with Women of Color : An Empowerment Perspective, Soc. Work, 35, 149-153, 1990.

Hagen, E. : Open mapping from the ground up : learning from Map Kibera, Making All Voices Count Research Report, IDS, Brighton, 2017.

Harley, J. B. : Deconstructing The Map, Cartographica : The International Journal for Geographic Information and Geovisualization, 26, 1-20, 1989.

Harris, T. and Weiner, D. : Empowerment, Marginalization, and "Community-integrated" GIS, Cartogr. Geogr. Inf. Sc., 25, 6776, 1998.

Harvey, D. : Social Justice and the City, Edward Arnold, London, 1973.

Hirt, I. : Redistribuer les cartes : Approche postcoloniale d'un processus de cartographie participative en territoire mapuche (Chili) $\mathrm{PhD}$, Faculté des Sciences Economiques et Sociales, Université de Genève, Genève, 477 pp., 2008.

Hirt, I. and Lerch, L. : Cartographier les territorialités indigènes dans les Andes boliviennes : enjeux politiques, défis méthodologiques, Cybergeo : European Journal of Geography, 638, https://doi.org/10.4000/cybergeo.25843, 2013.

Huggan, G. : Decolonizing the Map : Post-Colonialism, PostStructuralism and the Cartographic Connection, Ariel-Rev. Int. Engl., 20, 115-131, 1989.

Jacobs, J. M. : Edge of empire : postcolonialism and the city, Routledge, London, New York, 1996.

Lefebvre, H. : La production de l'espace, L'Homme et la société, 31-32, 15-32, 1974.

Lerch, L. : Mondialisation et digitalisation des territoires indigènes : rôles de l'aide internationale et des technologies de l'informations géographique dans les politiques du territoire et du développement en Bolivie, Ph.D. thesis, University of Geneva, Geneva, 2014.

Lukes, S. : Power, a radical view, Macmillan, London ; New York, 1974.

Mbembe, A. : Qu'est-ce que la pensée postcoloniale ?, Esprit, 330, 117-133, 2006

Mukherjee, F. : Public Participatory GIS, Geography Compass, 9, 384-394, 2015.

Nora, F. and Niina, K. : Participatory mapping and geographical patterns of the social landscape values of rural communities in Zanzibar, Tanzania, Fennia - International Journal of Geography, 187,2009

Odendaal, N. : Information and communication technology and urban transformation in South African cities, PhD, Faculty of Engineering and the Built Environment, University of Witwatersrand, Johannesburg, 2010.
Oldfield, S. and Greyling S. : Waiting for the state : a politics of housing in South Africa, Environment and Planning A : Economy and Space, 47, 1100-1112, https://doi.org/10.1177/0308518x15592309, 2015.

Pánek, J. : Participatory and Public Participation GIS : A Phenomenon of Neocartography with a High Potential in Developing Countries, in : The Scale of Globalization. Think Globally, Act Locally, Change Individually in the 21st Century, University of Ostrava, Ostrava, Czech Republic, 8-9 September 2011, 235243, 2011.

Parnell, S. and Pieterse, E. : Africa's Urban Revolution, Zed Books, London, 2014.

Peluso, N. L. : Whose Woods Are These ? Counter-mapping Forest Territories In Kalimantan, Indonesia, Antipode, 27, 383406, 1995.

Pickles, J. : Ground Truth 1995-2005, T. Gis., 10, 763-772, 2006.

Radil, S. M. and Anderson, M. B. : Rethinking PGIS : Participatory or (post)political GIS ?, Prog. Hum. Geog., 43, 195-213, 2019.

Republic of South Africa : White Paper on Local Government, Government Printers, Pretoria, 1998

Reynaud, A. : Société, espace et justice. Inégalités régionales et justice socio-spatiale, Presses Universitaires de France, Paris cedex 14,1981

Ricker, B., Cinnamon, J., and Dierwechter, Y. : When open data and data activism meet : An analysis of civic participation in Cape Town, South Africa, The Canadian Geographer / Le Géographe canadien, 1-15, https://doi.org/10.1111/cag.12608, 2020.

Robinson, J. : Ordinary cities : between modernity and development, Routledge, London, New York, 2006.

Roy, A. : Slumdog Cities : Rethinking Subaltern Urbanism, Int. J. Urban Regional, 35, 223-238, 2011.

Roy, A. : Who's Afraid of Postcolonial Theory ?, Int. J. Urban Regional, 40, 200-209, 2016.

Said, E. W. : Orientalism, Pantheon Books, New York, 1978.

Schurr, C. and Segebart, D. : Engaging with feminist postcolonial concerns through participatory action research and intersectionality, Geogr. Helv., 67, 147-154, https://doi.org/10.5194/gh-67147-2012, 2012.

Scott, J. C. : Weapons of the Weak Everyday Forms of Peasant Resistance, Yale University Press, New Haven, London, 1985.

Scott, J. C. : Seeing Like a State How Certain Schemes to Improve the Human Condition Have Failed, Yale University Press, New Haven, London, 1998.

Sen, A. : Development as Freedom, Oxford University Press, New York, 1999.

Soja, E. W. : The city and spatial justice, justice spatiale | spatial justice, 1, 1-5, 2009.

Solomon, B. B. : Empowerment : Social work in oppressed communities, J. Soc. Work Pract., 2, 79-91, 1987.

Spivak, G. C. : Can the Subaltern Speak ?, Die Philosophin, 14, 42-58, 1988.

Thompson, M. M. : Upside-Down GIS : The Future of Citizen Science and Community Participation, The Cartographic Journal, 53, 326-334, 2016.

Townley, G., Pearson, L., Lehrwyn, J. M., Prophet, N. T., and Trauernicht, M. : Utilizing Participatory Mapping and GIS to Examine the Activity Spaces of Homeless Youth, Am. J. Commun. Psychol., 57, 404-414, 2016. 
Turnbull, D. : Masons, Tricksters and Cartographers : Comparative Studies in the Sociology of Scientific and Indigenous Knowledge, Harwood Academic, Taylor and Francis, London, 2000.

UNHabitat : Habitat III : New Urban Agenda, United Nations Habitat, Nairobi, 66 pp., 2017.

Willis, K. : Informal Settlements. In : International Encyclopedia of Human Geography (Second Edition), edited by : Kobayashi, A., Elsevier, Oxford, 2020.
Young, C. J. : Refining a Conceptual Basemap : Critical GIS and Political Theory, Master of Arts, Department of Geography, University of Washington, Seattle, WA, 138 pp., 2012.

Zimmerman, M. A. : Empowerment theory : Psychological, organizational, and community levels of analysis, in : Handbook of community psychology, Kluwer Academic Publishers, Dordrecht, Netherlands, 2000. 